\title{
How much are 2d Yukawa models similar to the Gross-Neveu models?*
}

\author{
A.K. De ${ }^{\mathrm{a}}$, E. Focht ${ }^{\mathrm{b}, \mathrm{c}}$, W. Franzki ${ }^{\mathrm{b}, \mathrm{c}}$ and J. Jersák ${ }^{\mathrm{bc}}$

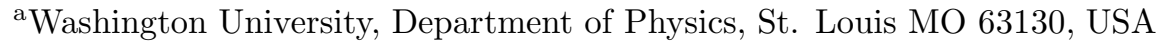 \\ ${ }^{\mathrm{b}}$ Institute for Theoretical Physics E, RWTH Aachen, Sommerfeldstr., 5100 Aachen, Germany \\ ${ }^{\text {c} H L R Z ~ c / o ~ K F A ~ J u ̈ l i c h, ~ P . O . ~ B o x ~ 1913, ~} 5170$ Jülich, Germany
}

We present numerical evidence that the $2 \mathrm{~d}$ Yukawa models with strong quartic selfcoupling of the scalar field have the same phase structure and are asymptotically free in the Yukawa coupling like the Gross-Neveu models.

\section{Yukawa models in 2 dimensions}

The 2d Yukawa models $\left(\mathrm{Y}_{2}\right)$ with chiral $\mathrm{Z}(2)$ or $\mathrm{U}(1)$ symmetries are natural extensions of the usual or chiral 2d Gross-Neveu (GN) models, respectively. Starting from the auxiliary field representation of the 4 -fermion coupling, one can add both the kinetic term and a self-interaction of this field $\phi$ into the GN action. On the lattice the $\mathrm{Z}(2)$ symmetric $\mathrm{Y}_{2}$ action is then (we introduce $N_{F} / 4$ "naive" Dirac fermion fields $\psi^{\alpha}$ )

$$
\begin{aligned}
S & =-2 \kappa \sum_{x, \mu} \phi_{x} \phi_{x+\mu}+\sum_{x} \phi_{x}^{2}+\lambda \sum_{x}\left(\phi_{x}^{2}-1\right)^{2} \\
& +\sum_{x, \alpha} \bar{\psi}_{x}^{\alpha} \not \partial \psi_{x}^{\alpha}+y \sum_{x, \alpha} \bar{\psi}_{x}^{\alpha} \phi_{x} \psi_{x}^{\alpha} .
\end{aligned}
$$

The $\phi^{4}$ scalar selfcoupling has been chosen, out of many possibilities in $2 \mathrm{~d}$, for the sake of analogy with the $4 \mathrm{~d}$ theories. For $\kappa=\lambda=0$ the $\mathrm{Z}(2)$ GN model is obtained if the Yukawa coupling $y$ is related to the usual GN coupling $g$ by $y=\sqrt{2} g$.

By choosing the above hopping parameter $\kappa$ formulation of the scalar field sector the kinetic term can be turned on or off gradually, elucidating the smoothness of the transition from the auxiliary to a dynamical field. The relationship

$y_{0}=\frac{y}{a \sqrt{2 \kappa}}$

between the Yukawa coupling $y_{0}$ used in continuum and $y$ is singular at $\kappa=0$, however. Another

\footnotetext{
*Talk presented by E. Focht at the "Lattice '92" conference, Amsterdam.
}

virtue of the hopping parameter formulation is that spin models with the $\mathrm{Z}(2)$ or $\mathrm{U}(1)$ symmetry (the Ising or the XY models, respectively) are easily recovered at $y=0$ and $\kappa$ finite by choosing $\lambda=\infty$. The $\mathrm{Y}_{2}$ models thus interpolate between the GN and spin models.

\section{Expected scaling properties}

Motivated by the recent discussion of a relationship between the Nambu-Jona-Lasinio type four-fermion theories and the Standard Model 113] we address here the question in which regions of the parameter space the $\mathrm{Y}_{2}$ models still possess the most cherished properties of the GN models, namely the asymptotic freedom at $y \rightarrow 0$, the fermion mass generation and, in the case of the $\mathrm{Z}(2)$ model, the symmetry breaking [4.5.5. In the GN models these properties are derived by means of the $1 / N_{F}$ expansion.

For $\mathrm{Y}_{2}$ models this expansion is applicable for $\lambda=\mathrm{O}\left(1 / N_{F}\right)$ [1.2] and here the same results as for the GN models are found. In particular, as long as $\kappa<\kappa_{c}(\lambda=0)=1 / 4$, the fermion mass $a m_{F}$ is expected to scale as

$a m_{F} \propto \exp \left[-\frac{1}{2 \beta_{0}} \frac{a^{2} m^{2}}{Z} \frac{1}{y^{2}}\right]$.

For $\lambda=0$ we have

$m^{2}=m_{0}^{2}=\left(1-\frac{\kappa}{\kappa_{c}(0)}\right) \frac{1}{a^{2} \kappa}, \quad Z=\frac{1}{2 \kappa}$,

with $m_{0}$ being the scalar field mass at $\lambda=y=0$. 
For large $\lambda$ the $1 / N_{F}$ expansion is à priori not applicable, not to speak of the perturbation theory. Nevertheless, M.A. Stephanov suggested [6] that in this case the mean field (MF) theory could be a good guide. An observation of long range ferromagnetic couplings in the effective scalar field theory at $y>0$ by E. Seiler [7] supports the applicability of the MF theory. The resulting expectations are [8]:

(i) The $\mathrm{Y}_{2}$ models at $\lambda=\infty$ or large possess at arbitrarily small $y$ only the broken symmetry (in the $\mathrm{Z}(2)$ case) or the spin-wave (in the $\mathrm{U}(1)$ case) phase, whereas the symmetric or vortex phase present in the $y=0$ case in the pure scalar theory is absent at $y>0$.

(ii) The fermion mass and the magnetization $y\langle\phi\rangle$ scale according to eq. (3). The coefficient $m^{2}$ is for $\kappa>0$ the squared scalar mass at $y=0$ and $Z$ its wave function renormalization constant. In general, $Z / m^{2}$ could be replaced by the scalar field propagator at zero momentum, i.e. the susceptibility. This should hold for any $\kappa<\kappa_{c}(\lambda)$ where $\kappa_{c}(\lambda)$ is the line of critical points in the pure scalar theory at $y=0$.

Thus according to the MF theory the asymptotic freedom and the other mentioned properties of the GN models might occur also for large $\lambda$ in the $\mathrm{Y}_{2}$ models.

\section{Methods of data analysis}

We have tried three methods:

(i) The asymptotic scaling law, which could be used to fit the data, is as (3) multiplied by $y^{-\beta_{1} / \beta_{0}^{2}}$. Here $\beta_{0}$ and $\beta_{1}$ are the $\beta$-function coefficients which in the GN limit have the perturbative values 9,10$]$

$\beta_{0}=\frac{N_{F}-1}{2 \pi} \quad, \quad \beta_{1}=-\frac{N_{F}-1}{(2 \pi)^{2}}$

in the model with $\mathrm{Z}(2)$ symmetry and

$\beta_{0}=\frac{N_{F}}{2 \pi} \quad, \quad \beta_{1}=-\frac{N_{F}}{2 \pi^{2}}$

in the $\mathrm{U}(1)$ case. This method does not take the finite volume effects into account. Nevertheless, one can roughly determine the values of the coefficient $\beta_{0}$ and compare with the values (5) and
(6). The 2-loop contribution given by $\beta_{1}$ is less important than the finite size effects.

(ii) A more appropriate way of analyzing the data is provided by a modified gap equation on finite lattices,

$\frac{a^{2} m^{2}}{Z} \frac{1}{y^{2}}=\frac{\pi \beta_{0}}{V} \sum_{\{p\}} \frac{1}{\sum_{\mu} \sin ^{2} p_{\mu}+\left(a m_{F} / s\right)^{2}}$

where the sum is performed over the fermion momenta with one periodic and one antiperiodic boundary condition on the $L^{2}$ lattices. Thanks to the IR divergence it leads for small $a m_{F}$ in the infinite volume limit to the scaling law (3).

The coefficient $\beta_{0}$ is considered as a free parameter in order to allow for its possible deviation from the perturbative value. The parameter $s$ takes into account the fact that the gap equation determines the value of the mass gap with insufficient precision. In order to fit the data it is necessary to treat the mass gap as a free parameter.

It would be interesting to determine the mass gap and compare it with the recently obtained exact results for the GN models [11,12]. With our present accuracy it could be only estimated to be roughly consistent with these results.

We note that one could take the finite size effects into account also beyond the leading $1 / N_{F}$ order [13, 14]. As we do not expect this expansion to be applicable for large $\lambda$, we do not attempt such refinements.

Once the parameters in the gap equation have been determined by a fit, the quantity $h(\kappa, \lambda)$,

$h(\kappa, \lambda)=\frac{1}{2 \beta_{0}} \frac{a^{2} m^{2}}{Z} \Rightarrow a m_{F} \propto \exp \left[-\frac{h(\kappa, \lambda)}{y^{2}}\right]$

has been extracted.

(iii) We have tried to fit the data also by an alternative to the essential singularity at $y=0$, namely by a hypothetical power law behavior

$m_{F}=a\left(y-y_{c}\right)^{b}$.

\section{Results at $\lambda=0$}

To gain experience we have first performed simulations at $\lambda=0$ in the interval $-0.1 \leq \kappa<$ $\kappa_{c}(\lambda=0)=1 / 4$. We have determined in both 
models the $y$-dependence of $a m_{F}$ and of $y\langle\phi\rangle$ in the $\mathrm{Z}(2)$ model at fixed values of $\kappa$ on lattices of size $16^{2}-64^{2}$. The following observations are useful for the study of the models at large $\lambda$ :

(i) The data analysis by means of the asymptotic law (3) both without and with the 2-loop correction is possible if those points at small $y$, which show finite size effects, are excluded (fig. 1). However, the obtained values of $\beta_{0}$ change with lattice size, when lower values of $a m_{F}$ can be taken into account on larger lattices, so that one probably does not see the true asymptotic behaviour.

(ii) The gap equation (7) can describe the data obtained on different lattices consistently by means of one set of parameter values, including $\beta_{0}$ (fig. 1). The onset of finite size effects at small $a m_{F}$, as well as the general tendency of the data at large $y$ are well reproduced. This analysis is superior to that by means of (3).

(iii) The fermion masses in both models and $y\langle\phi\rangle$ in the $\mathrm{Z}(2)$ model behave in a very analogous way. The magnetization in the $\mathrm{U}(1)$ case is present on finite lattices, but it shows a significant size dependence consistent with its vanishing in the infinite volume. Thus we observe the dynamical fermion mass generation taking place in spite of the absence of spontaneous symmetry breaking in $2 \mathrm{~d}$ [5].

(iv) The $\kappa$-dependence is consistent with the expected one, eq. (位). This is demonstrated in fig. 2, where $h(\kappa, 0)$ is shown. The values of $\beta_{0}$ obtained at different $\kappa$ are for both models consistent with the values (5) and (5) within $10 \%$.

(v) The power law formula (9) can fit the data well for any given lattice size. The parameters $b$ and $y_{c}$ depend strongly on the lattice size, however. This makes an algebraic singularity less probable than the essential one, but a reliable exclusion of the former on the basis of data alone seems very difficult. So one should be cautious at large $\lambda$, when the analytic information on the type of singularity is less reliable than at $\lambda=0$.

\section{Results at large $\lambda$}

Going to large $\lambda$ we have simulated both models at $\lambda=0.5$ and the $\mathrm{U}(1)$ model at $\lambda=\infty$ in the interval $-0.3 \leq \kappa<\kappa_{c}(\lambda)$. The most important results are:

(i) The $y$-dependence of the fermion mass, including the onset of the finite size effects, is described by the gap equation (7) nearly as well as in the $\lambda=0$ cases. This is demonstrated in fig. 3 for the $\mathrm{U}(1)$ model at $\lambda=\infty$ and $\kappa=0$ on $16^{2}-64^{2}$ lattices.

(ii) The power law behaviour (9) is not excluded but, similarly to $\lambda=0$, disfavored by the strong dependence of $y_{c}$ and $b$ on the lattice size. However, the finite size scaling analysis based on the Ansatz (9) has yet to be done.

(iii) These facts lead us to the tentative conclusion that at large $\lambda$, including $\lambda=\infty$, only the broken symmetry $(\mathrm{Z}(2)$ model $)$ or spin wave (U(1) model) phase is present at arbitrary small $y$.

(iv) Except at $\kappa=0$, we do not yet have an independent determination of $m^{2} / Z$ for large $\lambda$. Therefore we cannot yet determine the values of $\beta_{0}$ and give the results in form of the values of the coefficient $h(\kappa, \lambda)$. As an example we show $h(\kappa, \infty)$ for the $\mathrm{U}(1)$ model in fig. 4 (here $\left.\kappa_{c}(\infty) \approx 0.56\right)$. We hope to determine $\beta_{0}$ at large $\lambda$ for various $\kappa$ in the near future. At present our estimates indicate that its values are roughly consistent with $\beta_{0}$ in the GN cases, eqs. (5) and (6).

Thus we have found some evidence that the $\mathrm{Y}_{2}$ models with $\mathrm{Z}(2)$ and $\mathrm{U}(1)$ chiral symmetries with a strong $\phi^{4}$ interaction behave for $\kappa<\kappa_{c}(\lambda)$ as the GN models of the same symmetry, in particular they are asymptotically free. The transition from the GN to the spin model universality classes takes place probably at small $y$ and $\kappa \approx \kappa_{c}(\lambda)$.

\section{Acknowledgements}

We thank E. Seiler and M.A. Stephanov for helpful suggestions and A. Hasenfratz, P. Hasenfratz, R. Lacaze, F. Niedermayer and M.M. Tsypin for valuable discussions. The calculations have been performed on the CRAY Y-MP of HLRZ Jülich. This work has been supported by Deutsches Bundesministerium für Forschung und Technologie and by Deutsche Forschungsgemeinschaft. 


\section{REFERENCES}

1. A. Hasenfratz, P. Hasenfratz, K. Jansen, J. Kuti and Y. Shen, Nucl. Phys. B365 (1991) 79.

2. J. Zinn-Justin, Nucl. Phys. B367 (1991) 105.

3. W.A. Bardeen, C.T. Hill and M. Lindner, Phys. Rev. D41 (1990) 1647.

4. D.J. Gross and A. Neveu, Phys. Rev. D10 (1974) 3235.

5. E. Witten, Nucl. Phys. B145 (1978) 110.

6. M.A. Stephanov, private communication.

7. E. Seiler, private communication.

8. A.K. De, E. Focht, W. Franzki, J. Jersák and M.A. Stephanov, in preparation.

9. W. Wetzel, Phys. Lett. B153 (1985) 297.

10. C. Destri, Phys. Lett. B 210 (1988) 173 und B $213(1988) 565$ (E).

11. P. Forgács, F. Niedermayer und P. Weisz, Nucl. Phys. B 367 (1991) 123; Nucl. Phys. B 367 (1991) 144.

12. P. Forgács, S. Naik und F. Niedermayer, Physics Letters B 283 (1992) 282.

13. L. Bélanger, R. Lacaze, A. Morel, N. Attig, B. Petersson und M. Wolff, Nucl. Phys. B340 (1990) 245.

14. M. Campostrini, G. Curci and P. Rossi, Nucl. Phys. B314 (1989) 467.

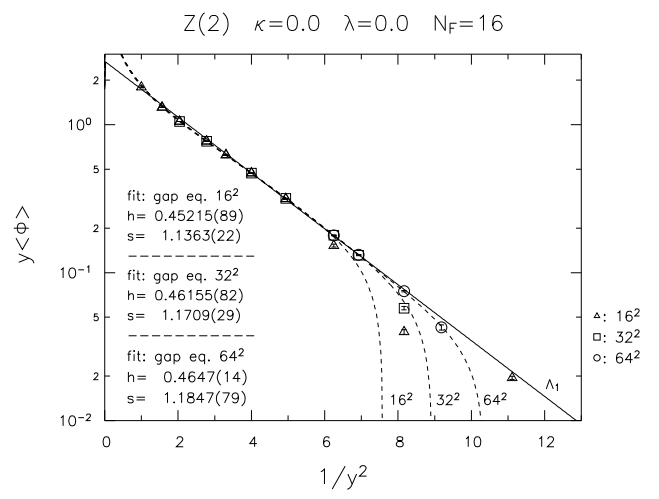

Figure 1. The $1 / y^{2}$ dependence of $y\langle\phi\rangle$ in the $Z$ (2) GN model $(\kappa=\lambda=0)$ with fits by eq. (17). The straight line is a fit by eq. (3).

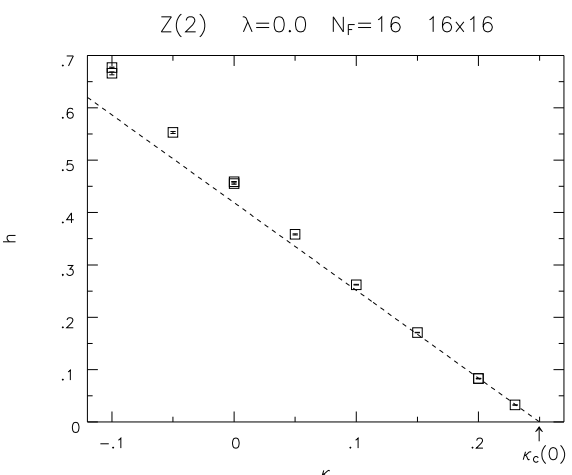

Figure 2. The $\kappa$ dependence of the coefficient $h(\kappa, 0)$ in the $Z(2)$ model at $\lambda=0$ is consistent with eq. (因) (dashed line).

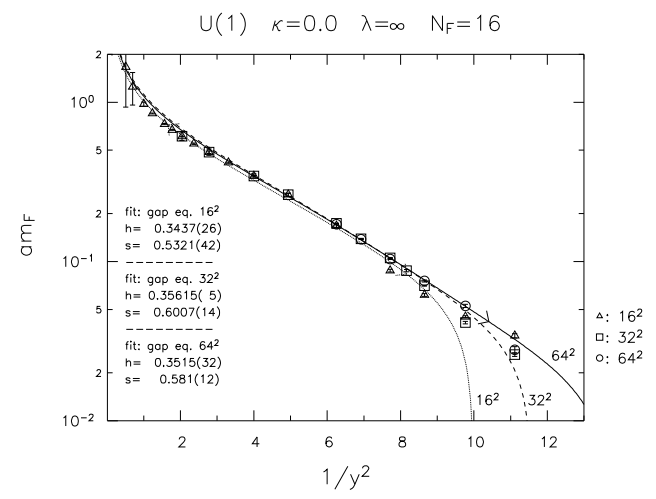

Figure 3. The $1 / y^{2}$ dependence of am $m_{F}$ in the $U(1) Y_{2}$ model with $N_{F}=16$ at $\lambda=\infty$ with fits by eq. (可.

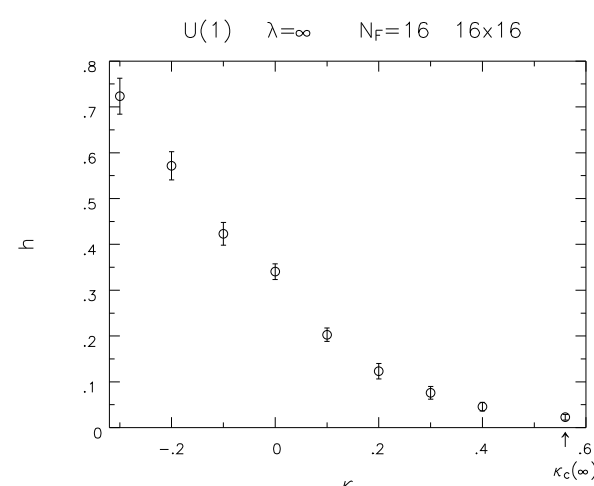

Figure 4. The $\kappa$ dependence of the coefficient $h(\kappa, \infty)$ in the $U(1)$ model at $\lambda=\infty$. 